protein allergy (CMPA). The clinical manifestations of this entity are diverse, thus establishing the diagnosis can be challenging. The aim of our human research is to evaluate and compare commonly performed diagnostic tests regarding CMPA.

Methods Children with symptoms suggesting cow's milk protein allergy were included in this study $(n=47)$. The investigation was performed at the Balassa János County Hosptital in Szekszárd, Hungary. Stool samples were collected from the participating children at the time of the diagnosis, and after 3 months of elimination diet. On the first hospital visit, blood samples were drawn for lymphocyte transformation test (LTT), and a skin allergy test was also performed. On the first and third visit, parents were asked to fill in a questionnaire about the child's symptoms, which was constructed by the research team.

Stool samples were analysed with a fecal calprotectin (FC) rapid test. The lymphocyte transformation tests were conducted in an accredited university laboratory. Skin allergy testing was performed in the hospital setting.

Evaluation of the data and the questionnaires was performed with SPSS statistical software.

Results In the study population ( $n=47$, mean age:7.36 years, $42.6 \%$ female), skin test was performed on 45 subjects; only 2 children (4.44\%) showed positive test result for cow's milk. Positive LTT was observed in 8 children (17\%), 4 subjects demonstrated questionable results.

Examining the entire research population, no significant difference in fecal calprotectin values was observed before (mean: $73.98 \mu \mathrm{g} / \mathrm{g}, \mathrm{SD}:$ 71.12) and after (mean: 68.11 $\mu \mathrm{g} / \mathrm{g}$, SD: 74.04) the elimination diet $(p=0.21)$. However, after dividing the participants into two subgroups according to the questionnaires, the following was observed: a significant decrease in FC values $(\mathrm{p}<0.001)$ was detected in children who strictly followed the diet $(n=35)$ comparing the first (mean: $84.057 \mu \mathrm{g} / \mathrm{g}, \mathrm{SD}:$ 79.48) and the second (mean: $41.114 \mu \mathrm{g} / \mathrm{g}$, SD: 34.24$)$ stool sample.

Conclusion According to our research data, skin allergy testing and LTT are not reliable diagnostic tools for establishing the diagnosis of CMPA. However, fecal calprotectin can be an objective parameter in confirming the diagnosis of allergic colitis in children with CMPA. Significant improvement in clinical symptoms can only be expected after a strictly followed elimination diet.

\section{GP182 ANTHROPOMETRIC INDICATORS AND BODY COMPOSITION IN CHILDREN WITH CEREBRAL PALSY}

Razilya Rakhmaeva*, Aelita Kamalova. Kazan State medical university, Kazan, Russian Federation

\subsection{6/archdischild-2019-epa.243}

Aim and objectives of the study Nutritional disorders are frequent in children with cerebral palsy (CP). The main methods for assessing the nutritional status of children with CP are the accurate interpretation of anthropometric indicators and the measures of body composition. We have started a study, which aims to identify features of physical development and body composition in children with $\mathrm{CP}$, depending on motor disabilities.

Patients and methods We assessed weight, height, BMI, tricipital skin fold thickness (TSFT), mid upper arm circumference
(MUAC) and body composition according to bioelectric impedance (BIA) in 34 children. The study included 39 patients (53.85\% boys and $46.15 \%$ girls) with spastic forms of CP aged 2 to 17 years. Patients were classified according to the form of CP and the results of GMFCS scale. The study group included children receiving physical rehabilitation, living in families, and feeding orally. Height, weight, and BMI were assessed using program WHO Anthro and WHO AnthroPlus.

Results In our study population 10/39 subjects (25.65\%) had GMFCS-I, 9/39 subjects (23.1\%) had GMFCS-II, 4/39 subjects had GMFCS-III (10.25\%), 14/39 subjects had GMFCS-IV (35.9\%), 2 subjects had GMFCS-V (5.1\%). The mean age was 9 y.o. Mean z-scores were -0.64 (SD 0.84) for weight, -1.17 (SD 0.98) for height, -1.17 (SD 1.1) for BMI, 0.54 (SD 0.62) for MUAC, and -0.29 (SD 0.74) for TSFT. The results of anthropometry revealed $24 / 39$ children with nutritional status disorders: among children with GMFCS-I - only 2/10 children (20\%), in children with GMFCS-II $-6 / 9$ children $(74.35 \%)$, in children with GMFCS-III $-3 / 4$ children (75\%), in children with GMFCS-IV - 11/14 children (78.6\%) and both of 2 children with GMFCS-IV (100\%). Focusing on body composition evaluated by BIA, an additional 7 children with altered body composition were revealed: $3 / 10-2 / 9-0 / 4-2 / 14$ accordingly GMFCS levels I-II-III-IV. Mean phase angle (PA) value was $5.5 \pm 0.39$ with value $<5.4$ in $13 / 39$ children $(33.3 \%)$.

Conclusion Nutritional disorders were revealed in 24/39 subjects $(61.5 \%)$ and $31 / 39$ subjects $(79.5 \%)$ with altered body composition in this sample of patients with CP. The frequency of detection of nutritional disorders in children with $\mathrm{CP}$ is higher in the group with more severe motor disability (GMFCS-IV > GMFCS-II > GMFCS-I). According to the results of BIA, decreased ACM level and PA value are the most sensitive indicators of nutritional status disorders.

\section{GP183 ACHIEVING THE PANACEA IN DIAGNOSING PAEDIATRIC AUTOIMMUNE PANCREATITIS - A CASE STUDY}

Graham King*, Emer O'Toole, Kevin O'Hare, Barbara Ryan, Basil El Nazir, Shoana Quinn. Tallaght University Hospital, Dublin, Ireland

\subsection{6/archdischild-2019-epa.244}

Introduction Both in the Republic of Ireland and worldwide paediatric autoimmune pancreatitis (P-AIP) is very rare and its incidence is largely unknown. To date, a few anecdotal cases in Ireland have been diagnosed histopathologically following resection of a pancreatic mass.

Case Report We report the case of a 13 yo boy admitted to the paediatric ward with a 4 day history of jaundice and pruritus, and a 2 day history of pale loose stools, dark urine, and vomiting. On examination he also had right upper quadrant abdominal tenderness. His liver profile showed biochemical signs of obstructive jaundice and cholestasis. He had a normal amylase and only mildly raised lipase. Total immunoglobulin level and IgG4 were normal.

Transabdominal ultrasound showed a distended gallbladder and a dilated CBD of $15 \mathrm{~mm}$. MRCP showed a possible $3 \mathrm{~cm}$ mass in the head of pancreas (HOP) with a normal pancreatic duct.

Our paediatric unit is within an adult hospital with subspecialist pancreatobiliary (PB) expertise including endoscopic ultrasound (EUS). EUS showed that the entire pancreas was expanded and no discrete mass was seen in the HOP. A EUS- 